\title{
GLOBAL RECONSTRUCTION OF NONLINEAR SYSTEMS FROM FAMILIES OF LINEAR SYSTEMS
}

\author{
D.J.Leith $^{1,2}$, W.E.Leithead ${ }^{1,2}$ \\ ${ }^{1}$ Hamilton Institute, NUI Maynooth, Co. Kildare, Ireland \\ ${ }^{2}$ Department of Electronic \& Electrical Engineering, \\ University of Strathclyde, 50 George St., Glasgow, U.K.
}

\begin{abstract}
This note concerns a fundamental issue in the modelling and realisation of nonlinear systems; namely, whether it is possible to uniquely reconstruct a nonlinear system from a suitable collection of transfer functions and, if so, under what conditions. It is established that a family of frozen-parameter linearisations may be associated with a class of nonlinear systems to provide an alternative realisation of such systems. Nevertheless, knowledge of only the input-output dynamics (transfer functions) of the frozen-parameter linearisations is insufficient to permit unique reconstruction of a nonlinear system. Under mild structural conditions, it is shown that knowledge of a family of augmented transfer functions is sufficient to permit a large class of nonlinear systems to be uniquely reconstructed. Essentially, the augmented family embodies the information necessary to select state-space realisations for the linearisations which are compatible with one another and with the underlying nonlinear system. Copyright $($ C) 2002 IFAC
\end{abstract}

Keywords: Nonlinear systems, LPV Systems, Gain-Scheduling, System Identification.

\section{INTRODUCTION}

This note concerns a fundamental issue in the modelling and realisation of nonlinear systems; namely, whether it is possible to uniquely reconstruct a nonlinear system from a suitable collection of transfer functions and, if so, under what conditions. Families of linear systems play an important role in many areas of nonlinear systems theory and practice. The construction of nonlinear systems related to a family of linear systems is, for example, the subject of the pseudo-linearisation (e.g. Reboulet \& Champetier 1984) and extended linearisation (e.g. Rugh 1986) approaches and plays a central role in the choice of realisation of gain-scheduled controllers (e.g. Leith \& Leithead 1998a). Families of linear systems also play an important role in system identification practice (e.g. McLoone \& Irwin 2000).

A key issue in many application domains is that the linear systems are specified only to within a linear state transformation; that is, the choice of state realisation is available as a degree of freedom. This is usually the situation, for example, in divide and conquer identification (because only input-output data is measurable) and many forms of gain-scheduling design (because the linear methods used to carry out point designs are generally insensitive to the choice of statespace realisation). The objective of this note is to investigate the conditions, if any, under which unique, global reconstruction of a nonlinear system is possible. In order to focus on structural factors and to improve the clarity of the development, attention is restricted here to situations where the linearisation family is wellposed and known exactly; that is, stochastic issues are considered outwith the scope of the present note.

\section{PRELIMINARIES}

It is well known that the family of classical perturbation linearisations of a nonlinear system need not fully characterise the dynamics of a nonlinear system. It is not possible to distinguish between systems having the same equilibrium dynamics but different dynamics away from equilibrium. For example, consider a family 
of equilibrium linearisations for which the member associated with the equilibrium operating point, $\left(r_{0}, x_{0}\right.$, $\mathrm{y}_{\mathrm{o}}$ ), is

$$
\begin{aligned}
& \delta \dot{\mathrm{x}}=-10.1 \delta \mathrm{x}+1.01 \delta \mathrm{r}, \quad \delta \mathrm{y}=\delta \mathrm{x} \\
& \delta r=r-r_{o}, \delta x=x-x_{o}, y=\delta y+y_{o}
\end{aligned}
$$

The linearised dynamics are the same at every equilibrium point and so might, for example, trivially be associated with the linear system

$$
\dot{\mathrm{x}}=-10.1 \mathrm{x}+1.01 \mathrm{r}, \mathrm{y}=\mathrm{x}
$$

However, it is straightforward to confirm that the linearised dynamics might equally be associated with any member of the family of nonlinear systems

$$
\dot{x}=G(r-10 x), \quad y=x
$$

for which $\mathrm{G}(\bullet)$ is any differentiable function such that $\nabla \mathrm{G}(0)=1.01$. To enable the nonlinear system to be reconstructed, it is necessary to adopt an approach which provides additional information about the dynamics of the system.

Borrowing notation from the LPV/quasi-LPV literature, consider systems of the form

$$
\begin{aligned}
& \dot{\mathbf{z}}=(\mathbf{A}+\phi(\boldsymbol{\theta}) \mathbf{M}) \mathbf{z}+(\mathbf{B}+\phi(\boldsymbol{\theta}) \mathbf{N}) \mathbf{u} \\
& \mathbf{v}=(\mathbf{C}+\varphi(\boldsymbol{\theta}) \mathbf{M}) \mathbf{z}+(\mathbf{D}+\varphi(\boldsymbol{\theta}) \mathbf{N}) \mathbf{u}
\end{aligned}
$$

where $\mathbf{u} \in \mathfrak{R}^{\mathrm{m}}, \mathbf{v} \in \mathfrak{R}^{\mathrm{p}}, \theta \in \mathfrak{R}^{\mathrm{q}}, \mathbf{z} \in \mathfrak{R}^{\mathrm{n}}, \phi, \varphi$ are nonlinear matrix functions and $\mathbf{A}, \mathbf{B}, \mathbf{C}, \mathbf{D}, \mathbf{M}, \mathbf{N}$ are appropriately dimensioned constant matrices. The defining characteristic of the systems in (4) is that the parameter variation enters via the nonlinear functions $\phi$, $\varphi$ which are, in turn, linearly coupled into the system equations through $\mathbf{M}, \mathbf{N}$. It is assumed that the "parameter" $\boldsymbol{\theta}$ is either measured directly or estimated from measurable signals but no restriction is otherwise placed on $\theta$. In particular, $\theta$ need not be an exogenous variable but may depend via a static or dynamic mapping on the state, $\mathbf{z}$, of the system. Confining attention to the class of systems (4) is not overly restrictive as it is easy to verify that any LPV/quasiLPV system can be formulated as in (4) by, if necessary, appropriately augmenting the parameter vector (trivially by including all the states and all the inputs when required).

Instead of the classical equilibrium linearisations, consider the family of linear systems with members

$$
\begin{aligned}
& \dot{\hat{\mathbf{z}}}=\left(\mathbf{A}+\phi\left(\theta_{1}\right) \mathbf{M}\right) \hat{\mathbf{z}}+\left(\mathbf{B}+\phi\left(\theta_{1}\right) \mathbf{N}\right) \mathbf{u} \\
& \hat{\mathbf{v}}=\left(\mathbf{C}+\varphi\left(\theta_{1}\right) \mathbf{M}\right) \hat{\mathbf{z}}+\left(\mathbf{D}+\varphi\left(\theta_{1}\right) \mathbf{N}\right) \mathbf{u}
\end{aligned}
$$

obtained by "freezing" the parameter, $\theta$, of the system (4). It is important to note that the frozen-parameter linearisation family includes information regarding not only the dynamics relating the input and output (characterised by the transfer function) but also the state-space realisation of each linearisation. As will become clearer in the sequel, the latter plays a key role in the reconstruction of the nonlinear dynamics from a family of frozen-parameter linearisations. Evidently, and quite unlike the situation with classical equilibrium linearisations, knowledge of the state-space frozenparameter linearisation family, (5), does completely define the nonlinear system (4) since it can be recovered by simply allowing $\theta$ to vary in (5); that is, the family of frozen-parameter linearisations is an alternative representation of the nonlinear system (4). Observe that, when $\theta$ depends on the state, $\mathbf{z}$, of the system, there is a frozen-parameter linearisation associated with every value of $\boldsymbol{\theta}$ even though in general some may only occur for off-equilibrium operating points. The restriction to near equilibrium operation inherent in the use of classical equilibrium linearisations is thereby avoided. Moreover, expanding, with respect to time, the solution $\mathbf{z}(\mathrm{t})$ of the system (4) relative to an initial time, $\mathrm{t}_{1}$,

$$
\mathbf{z}(\mathrm{t})=\mathbf{z}\left(\mathrm{t}_{1}\right)+\dot{\mathbf{z}}\left(\mathrm{t}_{1}\right) \delta \mathrm{t}+\varepsilon_{\mathrm{z}}
$$

with $\varepsilon_{\mathrm{z}}=\mathbf{z}(\mathrm{t})-\left\{\mathbf{z}\left(\mathrm{t}_{1}\right)+\dot{\mathbf{z}}\left(\mathrm{t}_{1}\right) \delta \mathrm{t}\right\}$, $\dot{\mathbf{z}}\left(\mathrm{t}_{1}\right)=\left(\mathbf{A}+\phi\left(\theta_{1}\right) \mathbf{M}\right) \mathbf{z}\left(\mathrm{t}_{1}\right)+\left(\mathbf{B}+\phi\left(\boldsymbol{\theta}_{1}\right) \mathbf{N}\right) \mathbf{u}\left(\mathrm{t}_{1}\right), \quad \delta \mathrm{t}=\mathrm{t}-\mathrm{t}_{1}$ and $\theta_{1}=\theta\left(\mathbf{z}\left(\mathrm{t}_{1}\right), \mathbf{N u}\left(\mathrm{t}_{1}\right)\right)$. Similarly, expanding the solution of the corresponding frozen-parameter linearisation, $\hat{\mathbf{z}}$, relative to time $t_{1}$ then

$$
\hat{\mathbf{z}}(\mathrm{t})=\hat{\mathbf{z}}\left(\mathrm{t}_{1}\right)+\dot{\hat{\mathbf{z}}}\left(\mathrm{t}_{1}\right) \delta \mathrm{t}+\varepsilon_{\hat{\mathbf{z}}}
$$

with $\dot{\hat{\mathbf{z}}}=\left(\mathbf{A}+\phi\left(\boldsymbol{\theta}_{1}\right) \mathbf{M}\right) \hat{\mathbf{z}}\left(\mathrm{t}_{1}\right)+\left(\mathbf{B}+\phi\left(\theta_{1}\right) \mathbf{N}\right) \mathbf{u}\left(\mathrm{t}_{1}\right)$. For initial condition $\hat{\mathbf{z}}\left(\mathrm{t}_{1}\right)=\mathbf{z}\left(\mathrm{t}_{1}\right)$, it can therefore be seen that the solution to (5) approximates the solution to (4) with error $\mathrm{O}\left(\delta \mathrm{t}^{2}\right)$; that is, to first-order in time. By combining the solutions to the members of the frozenparameter linearisation family in an appropriate manner, a global approximation to $\mathbf{z}(\mathrm{t})$ can be obtained. Over any time interval, $\left[\mathrm{t}_{1}, \mathrm{t}_{2}\right]$, an approximation is obtained by partitioning the interval into a number of short sub-intervals. Over each sub-interval, the approximate solution is the solution to (5) with $\theta_{1}$ equal to the value of $\theta$ at the operating point reached at the initial time for the sub-interval (with the initial conditions chosen to ensure continuity of the approximate solution). The approximation error over each sub-interval is proportional to the duration of the sub-interval squared. Hence, as the number of subintervals increases the number of local solutions pieced together increases, the approximation error associated with each decreases more quickly and the overall approximation error reduces. Indeed, since this construction is just Euler integration, it is straightforward to confirm that the overall approximation error tends to zero as the number of subintervals becomes unbounded.

\section{CONVENTIONAL TRANSFER FUNCTION ${ }^{1}$ KNOWLEDGE ALONE IS INSUFFICIENT}

The family of frozen-parameter linearisations, (5), completely defines the system (4) since it can be recovered by simply allowing $\theta$ to vary in (5); that is, the family of frozen-parameter linearisations is an alternative representation of the nonlinear system (4). Nevertheless, this equivalence is dependent on knowledge of the appropriate state co-ordinates for the frozen-parameter linearisations. For example, consider a system in the quasi-LPV form

$$
\begin{aligned}
& \dot{\mathbf{x}}=\mathbf{A}(\boldsymbol{\theta}) \mathbf{x}+\mathbf{B}(\boldsymbol{\theta}) \mathbf{r} \\
& \mathbf{y}=\mathbf{C}(\boldsymbol{\theta}) \mathbf{x}+\mathbf{D}(\boldsymbol{\theta}) \mathbf{r}
\end{aligned}
$$

with $\theta=\theta(\mathbf{x}, \mathbf{r})$. It can be seen immediately that any quasi-LPV system

\footnotetext{
${ }^{1}$ Throughout this paper the term 'transfer function' is used as shorthand to denote a linear model based only on measurable input-output data since this is the situation generally encountered in, for example, system identification and gainscheduling contexts. It includes, in addition to actual transfer function models, linear state-space models where the choice of state co-ordinates is only defined to within a linear transformation. No restriction to frequency-domain methods is implied or necessary.
} 


$$
\dot{\widetilde{\mathbf{x}}}=\mathrm{T}(\boldsymbol{\theta}) \mathbf{A}(\theta) \mathrm{T}^{-1}(\boldsymbol{\theta}) \widetilde{\mathbf{x}}+\mathrm{T}(\theta) \mathbf{B}(\theta) \mathbf{r}
$$$$
\tilde{\mathbf{y}}=\mathbf{C}(\theta) \mathrm{T}^{-1}(\theta) \tilde{\mathbf{x}}+\mathbf{D}(\theta) \mathbf{r}
$$

with $\mathbf{T}(\boldsymbol{\theta})$ non-singular, has frozen-parameter linearisations with transfer functions ${ }^{1}$ identical to those of (8). A system (9) may, of course, have quite different dynamics from those of (8): applying the state transformation $\overline{\mathbf{x}}=\mathbf{T}^{-1}(\boldsymbol{\theta}) \tilde{\mathbf{x}}$ yields

$$
\begin{aligned}
& \dot{\overline{\mathbf{x}}}=\mathbf{A}(\boldsymbol{\theta}) \overline{\mathbf{x}}+\mathbf{B}(\boldsymbol{\theta}) \mathbf{r}+\dot{\mathbf{T}}^{-1}(\boldsymbol{\theta}) \mathbf{T}(\boldsymbol{\theta}) \overline{\mathbf{x}} \\
& \tilde{\mathbf{y}}=\mathbf{C}(\boldsymbol{\theta}) \overline{\mathbf{x}}+\mathbf{D}(\boldsymbol{\theta}) \mathbf{r}
\end{aligned}
$$

Evidently, the dynamics, (10) (equivalent to (9)) differ from (8).

The impact of variations in $\mathrm{T}(\bullet)$ may also be seen in the context of constructing the solution to the nonlinear system from the piecewise combination of the solutions to the frozen-parameter linearisations (see §2). For example, consider the piecewise-linear system

$$
\dot{\mathbf{z}}=\mathbf{A}(\mathrm{t}) \mathbf{z}, \quad \mathbf{A}(\mathrm{t}) \in\left\{\mathbf{A}_{1}, \mathbf{A}_{2}, \ldots\right\}
$$

where $\mathbf{A}(\mathrm{t})=\mathbf{A}_{\mathrm{i}}$ on the interval $\left(\mathrm{t}_{\mathrm{i}}, \mathrm{t}_{\mathrm{i}-1}\right]$ with $\mathrm{t}_{1} \leq \mathrm{t}_{2} \leq \mathrm{t}_{3} \ldots$ and $\mathbf{A}_{\mathrm{i}}=\mathbf{T}_{\mathrm{i}} \mathbf{A} \mathbf{T}_{\mathrm{i}}^{-1}$. The solution may be written explicitly as

$$
\begin{aligned}
\mathbf{z}\left(\mathrm{t}_{\mathrm{k}}\right) & =\mathbf{e}^{\mathbf{A}_{k}\left(\mathrm{t}_{\mathrm{k}}-\mathrm{t}_{\mathrm{k}-1}\right)} \mathbf{e}^{\mathbf{A}_{k-1}\left(\mathrm{t}_{\mathrm{k}-1}-\mathrm{t}_{\mathrm{k}-2}\right)} \cdots \mathbf{e}^{\mathbf{A}_{1}\left(\mathrm{t}_{1}-\mathrm{t}_{\mathrm{o}}\right)} \mathbf{z}\left(\mathrm{t}_{\mathrm{o}}\right) \\
& =\mathbf{e}^{\mathbf{T}_{\mathrm{k}} \mathbf{A} \mathbf{T}_{\mathrm{k}}^{-1}\left(\mathrm{t}_{\mathrm{k}}-\mathrm{t}_{\mathrm{k}-1}\right)} \mathbf{e}^{\mathbf{T}_{\mathrm{k}-1} \mathbf{A} \mathbf{T}_{k-1}^{-1}\left(\mathrm{t}_{\mathrm{k}-1}-\mathrm{t}_{\mathrm{k}-2}\right)} \cdots \mathbf{e}^{\mathbf{T}_{\mathrm{o}} \mathbf{A} \mathbf{T}_{\mathrm{o}}^{-1}\left(\mathrm{t}_{1}-\mathrm{t}_{\mathrm{o}}\right)} \mathbf{z}\left(\mathrm{t}_{\mathrm{o}}\right)
\end{aligned}
$$

The solution is strongly dependent on the properties of the $\mathbf{T}_{\mathrm{i}}$. For example, when the $\mathbf{T}_{\mathrm{i}}$ are identical, the system is precisely linear and thus stable for A Hurwitz, whereas when the $\mathbf{T}_{\mathrm{i}}$ differ the system behaviour may be highly nonlinear and, in particular, unstable even when $\mathbf{A}$ is Hurwitz (e.g. with $\mathbf{A}(\mathrm{t}) \in\left\{\left[\begin{array}{cc}3.5 & -4.5 \\ 13.5 & -14.5\end{array}\right],\left[\begin{array}{cc}3.5 & 4.5 \\ -13.5 & -14.5\end{array}\right]\right\}$ the $\mathbf{A}_{\mathrm{i}}$ are Hurwitz and similar yet it is straightforward to confirm, e.g. by the results of Shorten \& Narendra (1998), that there exist switching sequences for which (11) is unstable).

The objective of the present paper is to study the situation where a nonlinear system (4) is to be reconstructed from the members of its frozen-parameter linearisations when the latter are specified only to within a linear state transformation (that is, only the transfer functions ${ }^{1}$ are specified and the choice of state realisation is an available as a degree of freedom). This is the situation, for example, in divide and conquer identification (because only input-output data is measurable, see for example McLoone \& Irwin 2000) and many forms of gain-scheduling design (because the linear methods used to carry out point designs are generally insensitive to the choice of state-space realisation, see for example Leith \& Leithead 2000). It is clear that, for each linear system, it is necessary to determine the appropriate choice of state which cannot be uniquely inferred from conventional transfer function information alone.

\section{CONDITIONS FOR RECONSTRUCTING A NONLINEAR SYSTEM}

It is evident from the foregoing discussion that additional information is required in order to permit a nonlinear system to be reconstructed in a unique manner from an associated family of linear transfer functions. Neither the conventional family of input/output transfer functions associated with the classical equilibrium linearisations nor the family of input/output transfer functions associated with the frozen-parameter linearisations satisfy this requirement.
The requirement is thus to determine a suitable family of linear state-space systems which both uniquely defines (to within a non-singular state transformation) a nonlinear system and which is, in turn, uniquely defined by its associated family of transfer functions.

4.1 Conditions for Uniqueness

Consider two nonlinear systems

$$
\begin{aligned}
& \dot{\mathbf{z}}=(\mathbf{A}+\phi(\boldsymbol{\theta}) \mathbf{M}) \mathbf{z}+(\mathbf{B}+\phi(\boldsymbol{\theta}) \mathbf{N}) \mathbf{u} \\
& \mathbf{v}=(\mathbf{C}+\varphi(\boldsymbol{\theta}) \mathbf{M}) \mathbf{z}+(\mathbf{D}+\varphi(\boldsymbol{\theta}) \mathbf{N}) \mathbf{u}
\end{aligned}
$$

and

$$
\begin{aligned}
& \dot{\widetilde{\mathbf{z}}}=(\tilde{\mathbf{A}}+\tilde{\phi}(\tilde{\boldsymbol{\theta}}) \tilde{\mathbf{M}}) \tilde{\mathbf{z}}+(\tilde{\mathbf{B}}+\tilde{\phi}(\tilde{\boldsymbol{\theta}}) \tilde{\mathbf{N}}) \mathbf{u} \\
& \tilde{\mathbf{v}}=(\tilde{\mathbf{C}}+\tilde{\varphi}(\tilde{\theta}) \tilde{\mathbf{M}}) \tilde{\mathbf{z}}+(\tilde{\mathbf{D}}+\tilde{\varphi}(\tilde{\boldsymbol{\theta}}) \tilde{\mathbf{N}}) \mathbf{u}
\end{aligned}
$$

The system (13) may be reformulated as

$$
\begin{aligned}
& \dot{\mathbf{z}}=\mathbf{A} \mathbf{z}+\mathbf{B u}+\phi(\theta) \vartheta \\
& \mathbf{v}_{\text {aug }}=\left[\begin{array}{c}
\mathbf{v} \\
\vartheta
\end{array}\right]=\left[\begin{array}{c}
\mathbf{C} \\
\mathbf{M}
\end{array}\right] \mathbf{z}+\left[\begin{array}{l}
\mathbf{D} \\
\mathbf{N}
\end{array}\right] \mathbf{u}+\left[\begin{array}{c}
\varphi(\theta) \vartheta \\
0
\end{array}\right]
\end{aligned}
$$

and similarly for (14). Assume that the following conditions are satisfied

(i) the members of the frozen-parameter linearisations families corresponding to (15) are controllable, observable and $\left[\begin{array}{ll}\mathbf{M} & \mathbf{N}\end{array}\right]$ is full rank

(ii) $\phi\left(\theta_{\mathrm{o}}\right), \tilde{\phi}\left(\theta_{\mathrm{o}}\right), \varphi\left(\theta_{\mathrm{o}}\right), \tilde{\varphi}\left(\theta_{\mathrm{o}}\right)$ are equal to zero, for some value of $\theta$ equal to $\theta_{\mathrm{o}}$

(iii) there exist no non-zero solutions $\Delta, \mathbf{X}$ and $\mathbf{Y}$, satisfying

$$
\left[\begin{array}{cc}
\Delta \mathbf{A}-\mathbf{A} \Delta & \Delta \mathbf{B} \\
\mathbf{C} \Delta & \mathbf{0}
\end{array}\right]=\left[\begin{array}{l}
\mathbf{X} \\
\mathbf{Y}
\end{array}\right]\left[\begin{array}{ll}
\mathbf{M} & \mathbf{N}
\end{array}\right], \mathbf{M} \Delta=\mathbf{0}
$$

(iv) corresponding members of the frozen-parameter linearisation families (i.e. for which $\theta=\theta_{1}, \tilde{\theta}=\theta_{1}$ ) have, respectively, the same transfer function from $\mathbf{u}$ to $\mathbf{v}_{\text {aug }}$ and from $\mathbf{u}$ to $\tilde{\mathbf{v}}_{\text {aug }}$.

Condition (i) is a standard minimality condition from linear theory whilst condition (ii) removes the possible ambiguity regarding the linear component, if any, of $\phi, \tilde{\phi}, \varphi, \tilde{\varphi}$. Note, condition (iii) needs to be tested for only one member of the linearisation family since it is then automatically satisfied by the entire family. Condition (iv) requires that the transfer function relating the input, $\mathbf{u}$, to $\vartheta$ is known in addition to the transfer function relating $\mathbf{u}$ to $\mathbf{v}$. More information than was available in section 3 is thus available

Proposition (Uniqueness) Assume that conditions (i)(iv) are satisfied. Then the nonlinear systems (13) and (14) are identical (to within a constant linear state transformation); that is, under structural conditions (i)(iii) the transfer function information specified in condition (iv) uniquely defines a nonlinear system.

Proof It follows immediately from standard linear theory that when condition (iv) is satisfied $\widetilde{\mathbf{A}}+\tilde{\phi}\left(\theta_{1}\right) \tilde{\mathbf{M}}=\mathbf{T}\left(\theta_{1}\right)\left(\mathbf{A}+\phi\left(\theta_{1}\right) \mathbf{M}\right) \mathbf{T}^{-1}\left(\theta_{1}\right), \tilde{\mathbf{B}}+\tilde{\phi}\left(\theta_{1}\right) \tilde{\mathbf{N}}=\mathbf{T}\left(\theta_{1}\right)\left(\mathbf{B}+\phi\left(\theta_{1}\right) \mathbf{N}\right)$ $\widetilde{\mathbf{C}}+\tilde{\varphi}\left(\theta_{1}\right) \tilde{\mathbf{M}}=\left(\mathbf{C}+\varphi\left(\theta_{1}\right) \mathbf{M}\right) \mathbf{T}^{-1}\left(\theta_{1}\right), \quad \tilde{\mathbf{D}}+\tilde{\varphi}\left(\theta_{1}\right) \tilde{\mathbf{N}}=\mathbf{D}+\varphi\left(\theta_{1}\right) \mathbf{N}$ $\tilde{\mathbf{M}}=\mathbf{M T}^{-1}\left(\theta_{1}\right), \tilde{\mathbf{N}}=\mathbf{N}$

$$
\forall \theta_{1} \in \mathfrak{R}^{\mathrm{q}}
$$

where $\mathbf{T}\left(\boldsymbol{\theta}_{1}\right)$ is a non-singular linear state transformation (which may be different for each member of a linear family). Let $\mathbf{T}\left(\boldsymbol{\theta}_{\mathrm{o}}\right)$ be the identity matrix; this involves no loss of generality since, by (i), 
it can always be achieved by applying an appropriate constant linear state transformation. Then, owing to the minimality conditions (ii), it follows that $\tilde{\mathbf{A}}=\mathbf{A}, \quad \tilde{\mathbf{B}}=\mathbf{B}, \quad \tilde{\mathbf{C}}=\mathbf{C}, \quad \tilde{\mathbf{D}}=\mathbf{D}, \quad \tilde{\mathbf{M}}=\mathbf{M}, \quad \tilde{\mathbf{N}}=\mathbf{N}$. Hence,

$\left.\left[\begin{array}{cc}\Delta\left(\theta_{1}\right) \mathbf{A}-\mathbf{A} \Delta\left(\theta_{1}\right) & \Delta\left(\theta_{1}\right) \mathbf{B} \\ -\mathbf{C} \Delta\left(\theta_{1}\right) & \mathbf{0}\end{array}\right]+\left[\begin{array}{c}\Delta\left(\theta_{1}\right) \tilde{\phi}\left(\theta_{1}\right) \\ \mathbf{0}\end{array}\right]\left[\begin{array}{ll}\mathbf{M}\end{array}\right]=\left[\begin{array}{l}\left(\phi\left(\theta_{1}\right)-\tilde{\phi}\left(\theta_{1}\right)\right) \\ \left(\varphi\left(\theta_{1}\right)-\tilde{\varphi}\left(\theta_{1}\right)\right)\end{array}\right] \mathbf{M} \quad \mathbf{N}\right]$
$\mathbf{M} \Delta\left(\theta_{1}\right)=\mathbf{0}$
$\forall \boldsymbol{\forall} \in \mathfrak{R}^{\mathrm{q}}$

where $\Delta\left(\theta_{1}\right)=\mathbf{T}^{-1}\left(\boldsymbol{\theta}_{1}\right)$-I. Condition (iii) ensures that $\Delta\left(\boldsymbol{\theta}_{1}\right)=0, \mathbf{X}=0$ and $\mathbf{Y}=0$ is the only solution to

$\left[\begin{array}{cc}\Delta\left(\theta_{1}\right) \mathbf{A}-\mathbf{A} \Delta\left(\theta_{1}\right) & \Delta\left(\theta_{1}\right) \mathbf{B} \\ -\mathbf{C} \Delta\left(\theta_{1}\right) & \mathbf{0}\end{array}\right]=\left[\begin{array}{l}\mathbf{X} \\ \mathbf{Y}\end{array}\right]\left[\begin{array}{ll}\mathbf{M} & \mathbf{N}\end{array}\right], \mathbf{M} \Delta\left(\rho_{1}\right)=\mathbf{0}$

and so $\tilde{\phi}\left(\theta_{1}\right)=\phi\left(\theta_{1}\right), \tilde{\varphi}\left(\theta_{1}\right)=\varphi\left(\theta_{1}\right) \quad \forall \theta_{1} \in \mathfrak{R}^{\mathrm{q}}$ as

required. Consequently, under the foregoing conditions the nonlinear systems (13) and (14) must be identical to within a constant linear state transformation.

Remark: Genericity of condition (iii). Violation of condition (iii) requires the simultaneous satisfaction of many linear constraints. Specific systems violating condition (iii) do exist. Nevertheless, the class of linearisation families for which there exist non-zero solutions to (16) is non-generic. This can be seen as follows. The number of unknowns in (16) is $n^{2}+n q+p n$ while the number of linear equations is $n^{2}+n q+p n+n m$, where $\mathrm{n}, \mathrm{m}, \mathrm{p}, \mathrm{q}$ are the dimensions, respectively, of the state, input, output and parameter vectors. From standard linear theory, for any singular matrix there exists an arbitrarily small perturbation which makes it non-singular; that is, non-singular matrices are generic. When $\mathrm{m}$ is zero, the number of linear equations is the same as the number of unknowns and it follows immediately from the genericity of non-singular matrices that condition (iii) is also generically satisfied. When $\mathrm{m}$ is non-zero, violation of condition (iii) requires singularity of an $n^{2}+n q+p n$ subsets of equations subject to $\mathrm{nm}$ equality constraints and again genericity follows immediately. Hence, for most practical purposes condition (iii) may be assumed to always be satisfied (that is, except in singular circumstances where there exist specific applicationrelated constraints such that consideration of the nongeneric solutions to (16) is essential).

\section{$4.2 \theta$ and $\vartheta$ Linearly Related}

Condition (iv) in Section 4.1 requires knowledge of the transfer functions relating $\vartheta$ to the input $\mathbf{u}$. When $\vartheta$ is linearly related to $\theta$ and known a priori, the transfer function of one may be inferred from that of the other. In these circumstances, condition (iv) can be modified to a requirement for knowledge of the frozen-parameter transfer functions relating $\mathbf{u}$ to $\left[\begin{array}{l}\mathbf{v} \\ \boldsymbol{\theta}\end{array}\right]$. The Uniqueness Proposition may therefore be readily specialised as follows.

Corollary ( $\vartheta$ Linearly Related to $\theta$ ) When $\vartheta$ is linearly related to $\theta$, conditions (i)-(iii) of section 4.1 together with knowledge of the frozen-parameter transfer functions relating $\mathbf{u}$ to $\left[\begin{array}{l}\mathbf{v} \\ \boldsymbol{\theta}\end{array}\right]$ and the relationship between $\vartheta$ and $\theta$ uniquely defines a nonlinear system (4). (A similar situation pertains when, for example, the elements of $\theta$ are a subset the elements of $\vartheta$ or when the mapping from $\vartheta$ to $\theta$ is defined indirectly via some third quantity, $\xi$ say; note that $\xi$ may be measurable when $\theta$ and $\vartheta$ are not).

Remark: In this context Condition (iv) of the Uniqueness Condition is a very natural requirement. Information concerning the local evolution of the state is provided by the transfer function relating $\mathbf{u}$ to $\mathbf{v}$. However, the frozen linearisation evolves as the state evolves. Hence, to construct non-local solutions, the information is required to also update the member of the frozen linearisation family being used to define the evolution of the state. This additional information is provided by the transfer function relating $\mathbf{u}$ to $\theta$. Examples in the literature to which this corollary is directly relevant include: state-dependent systems (Priestley 1988), velocity-based systems (Leith \& Leithead 1998b,c).

\subsection{A Reconstruction Methodology}

In state-space terms, under conditions (i)-(iv) of section 4.1 the linear family with members

$$
\begin{aligned}
& \dot{\hat{\mathbf{z}}}=\mathbf{T}\left(\boldsymbol{\theta}_{1}\right) \hat{\mathbf{A}}\left(\boldsymbol{\theta}_{1}\right) \mathbf{T}^{-1}\left(\boldsymbol{\theta}_{1}\right) \hat{\mathbf{z}}+\mathbf{T}\left(\boldsymbol{\theta}_{1}\right) \hat{\mathbf{B}}\left(\boldsymbol{\theta}_{1}\right) \mathbf{u} \\
& {\left[\begin{array}{c}
\hat{\mathbf{v}} \\
\hat{\vartheta}
\end{array}\right]=\left[\begin{array}{c}
\hat{\mathbf{C}}\left(\boldsymbol{\theta}_{1}\right) \\
\hat{\mathbf{M}}\left(\boldsymbol{\theta}_{1}\right)
\end{array}\right] \mathbf{T}^{-1}\left(\boldsymbol{\theta}_{1}\right) \hat{\mathbf{z}}+\left[\begin{array}{c}
\hat{\mathbf{D}}\left(\boldsymbol{\theta}_{1}\right) \\
\hat{\mathbf{N}}
\end{array}\right] \mathbf{u}}
\end{aligned}
$$

is known but the state transformation, $\mathbf{T}(\bullet)$, relating the co-ordinates of one member to another is unknown. Note that ${ }^{\wedge}$ notation is used to emphasise the distinction between the frozen-parameter linearisations and the associated nonlinear system. Assume, without loss of generality, that $\mathbf{T}\left(\boldsymbol{\theta}_{\mathbf{0}}\right)=\mathbf{I}$ (recalling that the system is defined to within a constant linear state transformation, this assumption corresponds to one choice of linear transformation). Assume, also without loss of generality, that the constant matrices associated with the dynamics are

$\mathbf{A}=\hat{\mathbf{A}}\left(\theta_{\mathrm{o}}\right), \mathbf{B}=\hat{\mathbf{B}}\left(\theta_{\mathrm{o}}\right), \mathbf{C}=\hat{\mathbf{C}}\left(\boldsymbol{\theta}_{\mathrm{o}}\right), \mathbf{D}=\hat{\mathbf{D}}\left(\theta_{\mathrm{o}}\right), \mathbf{M}=\hat{\mathbf{M}}\left(\boldsymbol{\theta}_{\mathrm{o}}\right)(20)$ (this simply serves to fix any linear component of the system nonlinearity). The coefficients of the nonlinear system associated with (19) can be obtained as the solution, $\{\mathbf{T}(\bullet), \phi(\bullet), \varphi(\bullet)\}$, to the following linear equalities.

$\left[\begin{array}{cc}\mathbf{T}\left(\theta_{1}\right) \hat{\mathbf{A}}\left(\theta_{1}\right)-\mathbf{A T}\left(\theta_{1}\right) & \mathbf{T}\left(\theta_{1}\right) \hat{\mathbf{B}}-\mathbf{B}\left(\theta_{1}\right) \\ \hat{\mathbf{C}}\left(\theta_{1}\right)-\mathbf{C T}\left(\theta_{1}\right) & \hat{\mathbf{D}}\left(\theta_{1}\right)-\mathbf{D}\end{array}\right]=\left[\begin{array}{l}\phi\left(\theta_{1}\right) \\ \varphi\left(\theta_{1}\right)\end{array}\right]\left[\begin{array}{ll}\hat{\mathbf{M}}\left(\theta_{1}\right) & \mathbf{N}\end{array}\right]$

$\hat{\mathbf{M}}\left(\theta_{1}\right)-\mathbf{M T}\left(\theta_{1}\right)=0, \hat{\mathbf{N}}=\mathbf{N}$

A solution to (21) is guaranteed to be unique by the conditions in the foregoing proposition and corollaries; the nonlinear system thus reconstructed is described by

$$
\begin{aligned}
& \dot{\mathbf{z}}=(\mathbf{A}+\phi(\boldsymbol{\theta}) \mathbf{M}) \mathbf{z}+(\mathbf{B}+\phi(\boldsymbol{\theta}) \mathbf{N}) \mathbf{u} \\
& \mathbf{v}=(\mathbf{C}+\varphi(\boldsymbol{\theta}) \mathbf{M}) \mathbf{z}+(\mathbf{D}+\varphi(\boldsymbol{\theta}) \mathbf{N}) \mathbf{u}
\end{aligned}
$$

\section{SOME APPLICATIONS}

5.1 Extended local linear equivalence systems

Let $E=\left\{\mathbf{z}_{\mathrm{o}}, \mathbf{u}_{\mathrm{o}}: \mathbf{A} \mathbf{z}_{\mathrm{o}}+\mathbf{B} \mathbf{u}_{\mathrm{o}}+\phi\left(\theta\left(\mathbf{z}_{\mathrm{o}}, \mathbf{u}_{\mathrm{o}}\right)\right)\left[\mathbf{M \mathbf { z } _ { \mathrm { o } }}+\mathbf{N} \mathbf{u}_{\mathrm{o}}\right]=0\right\}$ denote the set of equilibrium points of the system (4), $\mathrm{R}_{\theta}(\mathrm{E})$ denote the range of $\theta$ on $\mathrm{E}$ (i.e. $\mathrm{R}_{\theta}(\mathrm{E})=\{\theta(\mathbf{z}, \mathbf{u})$ : $((\mathbf{z}, \mathbf{u}) \in \mathrm{E}\})$ and $\mathrm{R}_{\theta}(\Phi)$ the range of $\theta$ on the full operating space of the system, $\Phi=\left\{(\mathbf{z}, \mathbf{u}): \mathbf{z} \in \mathfrak{R}^{\mathrm{n}}\right.$, $\left.\mathbf{u} \in \mathfrak{R}^{\mathrm{m}}\right\}$. Systems, (4), for which

$$
\mathrm{R}_{\theta}(\mathrm{E})=\mathrm{R}_{\theta}(\Phi)
$$


are referred to here as extended local linear equivalence (ELLE) systems. The condition, (23), simply corresponds to the requirement that $\theta$ is parameterised by the equilibrium points. It follows immediately that the equilibrium information, $\left\{\left[\begin{array}{ll}\mathbf{A}+\phi(\boldsymbol{\theta}) \mathbf{M} & \mathbf{B}+\phi(\boldsymbol{\theta}) \mathbf{N} \\ \mathbf{C}+\boldsymbol{\varphi}(\boldsymbol{\theta}) \mathbf{M} & \mathbf{D}+\boldsymbol{\varphi}(\boldsymbol{\theta}) \mathbf{N}\end{array}\right]: \boldsymbol{\theta} \in \mathrm{R}_{\boldsymbol{\theta}}(\mathrm{E})\right\}$, together with knowledge of $\theta$, completely defines an ELLE system. In view of the importance of equilibrium information in classical theory (particularly gain-scheduling theory), and the relative ease with which equilibrium dynamics may be identified from measured data, the class of ELLE systems is of considerable interest in its own right. Note that even if not exactly satisfied, it is often possible to utilise, within a useful operating envelope, an ELLE approximation to a non-ELLE system. The results of section 4 can be immediately specialised to ELLE systems.

Example Wiener-Hammerstein system

Suppose that the frozen-parameter linearisation transfer functions relating $\mathbf{v}_{\text {aug }}$ and $\mathrm{u}$ are known and given by

$$
\mathbf{V}_{\text {aug }}(s)=\left[\begin{array}{c}
\frac{K\left(\theta_{1}\right)}{(s+a)(s+b)} \\
\frac{1}{(s+a)}
\end{array}\right] U(s)
$$

where $\mathbf{V}_{\text {aug }}(\mathrm{s}), \mathrm{U}(\mathrm{s})$ denote, respectively, the Laplace transforms of $\mathbf{v}_{\text {aug }}$, u. Assume also that the structure of the dynamics is such that $\theta$ equals $\vartheta$. Equivalently, in state-space terms, we have that

$$
\begin{aligned}
& \dot{\hat{\mathbf{z}}}=\mathbf{T}\left(\theta_{1}\right)\left[\begin{array}{cc}
-\mathrm{a} & 0 \\
\mathrm{~K}\left(\theta_{1}\right) & -\mathrm{b}
\end{array}\right] \mathbf{T}^{-1}\left(\theta_{1}\right) \hat{\mathbf{z}}+\mathbf{T}\left(\theta_{1}\right)\left[\begin{array}{l}
1 \\
0
\end{array}\right] \mathrm{u} \\
& \hat{\mathbf{v}}_{\text {aug }}=\left[\begin{array}{c}
\hat{\mathrm{V}} \\
\hat{\vartheta}
\end{array}\right]=\left[\begin{array}{ll}
0 & 1 \\
1 & 0
\end{array}\right] \mathbf{T}^{-1}\left(\theta_{1}\right) \hat{\mathbf{z}}
\end{aligned}
$$

where $\mathbf{T}(\bullet)$ is an unknown state transformation and the $\wedge$ notation is used to emphasise the distinction between the frozen-parameter linearisations and the associated nonlinear system. The linearisations, (25), are controllable and observable. Assume, without loss of generality, that $\mathbf{T}\left(\theta_{0}\right)=\mathbf{I}$ (recalling that the system is defined to within a global linear state transformation, this assumption corresponds to one choice of global linear transformation). Assume, also without loss of generality, that the constant matrices associated with the nonlinear dynamics are

$\mathbf{A}=\left[\begin{array}{cc}-\mathrm{a} & 0 \\ \mathrm{~K}\left(\theta_{\mathrm{o}}\right) & -\mathrm{b}\end{array}\right], \mathbf{B}=\left[\begin{array}{l}1 \\ 0\end{array}\right], \mathbf{C}=\left[\begin{array}{ll}0 & 1\end{array}\right], \mathrm{D}=0, \mathbf{M}=\left[\begin{array}{ll}1 & 0\end{array}\right], \mathrm{N}=0(26)$

(this simply serves to fix any linear component of the system nonlinearity). Uniqueness condition, (16), only requires to be evaluated for a single member of the linearisation family; taking the member corresponding to $\theta$ equal to $\theta_{\mathrm{o}}$ yields

$$
\left[\begin{array}{ccc}
\Delta_{12} \mathrm{~K}\left(\theta_{\mathrm{o}}\right) & (\mathrm{a}-\mathrm{b}) \Delta_{12} & \Delta_{11} \\
(\mathrm{~b}-\mathrm{a}) \Delta_{21}+\left(\Delta_{22}-\Delta_{11}\right) \mathrm{K}\left(\theta_{\mathrm{o}}\right) & -\Delta_{12} \mathrm{~K}\left(\theta_{\mathrm{o}}\right) & \Delta_{21} \\
\Delta_{21} & \Delta_{22} & 0
\end{array}\right]=\left[\begin{array}{ccc}
X_{1} & 0 & 0 \\
X_{2} & 0 & 0 \\
Y_{1} & 0 & 0
\end{array}\right](27)
$$

where $\Delta_{\mathrm{ij}}$ denotes the $\mathrm{ij}^{\text {th }}$ element of $\Delta$, and similarly for $\mathrm{X}_{\mathrm{i}}$. and $\mathrm{Y}_{1}$ Evidently, $\Delta=\mathbf{0}, \mathbf{X}=\mathbf{0}, \mathbf{Y}=\mathbf{0}$ is the sole solution, as required. Conditions (i)-(iv) are satisfied and it therefore follows from the foregoing proposition that (24) uniquely defines a nonlinear system. From (21), the coefficients of the nonlinear system associated with (24) (equivalently, (25)) are obtained as the solution to the following linear equalities (note that the existence of a unique solution is guaranteed by the foregoing conditions).

$\left.\left[\begin{array}{ll}\mathrm{T}_{11} & \mathrm{~T}_{12} \\ \mathrm{~T}_{21} & \mathrm{~T}_{22}\end{array}\right] \begin{array}{cc}-\mathrm{a} & 0 \\ \mathrm{~K}\left(\theta_{1}\right) & -\mathrm{b}\end{array}\right]-\left[\begin{array}{cc}-\mathrm{a} & 0 \\ \mathrm{~K}\left(\theta_{\mathrm{o}}\right) & -\mathrm{b}\end{array}\right]\left[\begin{array}{ll}\mathrm{T}_{11} & \mathrm{~T}_{12} \\ \mathrm{~T}_{21} & \mathrm{~T}_{22}\end{array}\right]=\left[\begin{array}{l}\phi_{1}\left(\theta_{1}\right) \\ \phi_{2}\left(\theta_{1}\right)\end{array}\right]\left[\begin{array}{ll}1 & 0\end{array}\right]$

$\left[\begin{array}{ll}0 & 1\end{array}\right]-\left[\begin{array}{ll}0 & 1\end{array}\right]\left[\begin{array}{ll}\mathrm{T}_{11} & \mathrm{~T}_{12} \\ \mathrm{~T}_{21} & \mathrm{~T}_{22}\end{array}\right]=\varphi\left(\theta_{1}\right)\left[\begin{array}{ll}1 & 0\end{array}\right]$

$\left[\begin{array}{ll}1 & 0\end{array}\right]-\left[\begin{array}{ll}1 & 0\end{array}\right]\left[\begin{array}{ll}\mathrm{T}_{11} & \mathrm{~T}_{12} \\ \mathrm{~T}_{21} & \mathrm{~T}_{22}\end{array}\right]=0,\left[\begin{array}{ll}\mathrm{T}_{11} & \mathrm{~T}_{12} \\ \mathrm{~T}_{21} & \mathrm{~T}_{22}\end{array}\right]\left[\begin{array}{l}1 \\ 0\end{array}\right]-\left[\begin{array}{l}1 \\ 0\end{array}\right]=0$

It is straightforward to verify that the solution to (28) is

$\mathbf{T}\left(\theta_{1}\right)=\left[\begin{array}{ll}\mathrm{T}_{11} & \mathrm{~T}_{12} \\ \mathrm{~T}_{21} & \mathrm{~T}_{22}\end{array}\right]=\left[\begin{array}{ll}1 & 0 \\ 0 & 1\end{array}\right] \phi\left(\theta_{1}\right)=\left[\begin{array}{c}0 \\ \mathrm{~K}\left(\theta_{1}\right)-\mathrm{K}\left(\theta_{\mathrm{o}}\right)\end{array}\right] \varphi\left(\theta_{1}\right)=0$

That is, the nonlinear system uniquely defined by the input-output information (24) is

$$
\begin{aligned}
& \dot{\mathbf{z}}=\left[\begin{array}{cc}
-\mathrm{a} & 0 \\
0 & -\mathrm{b}
\end{array}\right] \mathbf{z}+\left[\begin{array}{l}
1 \\
0
\end{array}\right] \mathrm{u}+\left[\begin{array}{c}
0 \\
\mathrm{~K}(\boldsymbol{\theta}) \vartheta
\end{array}\right] \\
& {\left[\begin{array}{l}
\mathrm{y} \\
\vartheta
\end{array}\right]=\left[\begin{array}{ll}
0 & 1 \\
1 & 0
\end{array}\right] \mathbf{z}}
\end{aligned}
$$

with $\theta=\vartheta$. This system is of Wiener-Hammerstein form. The frozen-parameter linearisation family is parameterised by the quantity, $\theta$, while the family of equilibrium points of (30) may be parameterised by the value of the input, $u$, at equilibrium. Since $\theta=u / a$ at equilibrium, the family of equilibrium points may therefore also be parameterised by $\theta$, and vice-versa. Hence, (30) belongs to the class of ELLE systems and, in accordance with the definition of this class, the frozen-parameter linearisation family (and so the global nonlinear dynamics) is completely defined by the family of frozen-parameter linearisations at the equilibrium points taken together with appropriate knowledge of $\theta$.

Remark Correspondence between equilibrium linearisations and frozen-parameter linearisations

In the particular situation where the frozen-parameter linearisations considered are, in fact, VB linearisations (Leith \& Leithead 1998b,c), a strong link can be established between the frozen-parameter linearisations and the classical equilibrium linearisations. For ELLE systems the VB frozen-parameter linearisation family can be determined directly, by inspection, from the classical equilibrium linearisations. It follows immediately that, for ELLE systems, the nonlinear dynamics can be uniquely reconstructed from the classical equilibrium linearisation family taken together with appropriate knowledge of the VB scheduling variable, $\rho$.

\subsection{Finite parameterisation of linearisation family by} blending local models.

The frozen-parameter linearisation family associated with a nonlinear system generally has infinitely many members. In many situations it is preferable to work with a small number of "representative" linearisations and recover the full linearisation family by blending or interpolating between these linearisations. Similar issues arise in many application domains and the literature on blended representations is extensive (see, for example, the survey by Johansen \& Murray-Smith 1997, Leith \& Leithead 1999, 2000), including numerous approaches related to gain-scheduling. A typical blended multiple model formulation of the nonlinear system (4) blends the linear local models 


$$
\begin{aligned}
& \dot{\mathbf{z}}=\left(\mathbf{A}+\phi\left(\boldsymbol{\theta}_{\mathrm{i}}\right) \mathbf{M}\right) \mathbf{z}+\left(\mathbf{B}+\phi\left(\boldsymbol{\theta}_{\mathrm{i}}\right) \mathbf{N}\right) \mathbf{u} \\
& \mathbf{v}=\left(\mathbf{C}+\varphi\left(\boldsymbol{\theta}_{\mathrm{i}}\right) \mathbf{M}\right) \mathbf{z}+\left(\mathbf{D}+\varphi\left(\boldsymbol{\theta}_{\mathrm{i}}\right) \mathbf{N}\right) \mathbf{u}
\end{aligned}
$$

together via the weighting functions $\mu_{\mathrm{i}} \mathrm{i}=1, \ldots$ to yield the nonlinear dynamics

$$
\begin{aligned}
& \dot{\mathbf{z}}=\sum_{\mathrm{i}}\left(\left(\mathbf{A}+\phi\left(\theta_{\mathrm{i}}\right) \mathbf{M}\right) \mathbf{z}+\left(\mathbf{B}+\phi\left(\theta_{\mathrm{i}}\right) \mathbf{N}\right) \mathbf{u}\right) \mu_{\mathrm{i}}(\theta) \\
& \mathbf{v}=\sum_{\mathrm{i}}\left(\left(\mathbf{C}+\varphi\left(\theta_{\mathrm{i}}\right) \mathbf{M}\right) \mathbf{z}+\left(\mathbf{D}+\varphi\left(\theta_{\mathrm{i}}\right) \mathbf{N}\right) \mathbf{u}\right) \mu_{\mathrm{i}}(\boldsymbol{\theta})
\end{aligned}
$$

Provided $\mu_{\mathrm{k}}\left(\theta_{\mathrm{j}}\right)$ is unity when $\mathrm{j}=\mathrm{k}$ and zero when $\mathrm{j} \neq \mathrm{k}$, the frozen-parameter linearisations of (32) corresponding to parameter value $\theta_{\mathrm{i}}$ is just the local model (31). Consider relaxing condition (iv) of the Uniqueness Proposition to the weaker requirement that the frozen-parameter transfer functions relating $\mathbf{u}$ to $\mathbf{v}_{\mathrm{aug}}$ are known only for parameter values $\left\{\boldsymbol{\theta}_{\mathrm{i}}, \mathrm{i}=1, \ldots \mathrm{N}\right\}$ (rather than for all parameter values). It follows directly from the proof of the Uniqueness Proposition that this relaxed condition (iv), together with conditions (i)-(iii) of section 4, uniquely defines the local models, (31). Compatible state-space realisations of the local models can be determined using the procedure described in the section 4.3 above. The blended nonlinear system, (32), is then defined by an appropriate choice of weighting functions $\mu_{\mathrm{i}}$ (for example, the use of triangular weighting functions corresponds to linear interpolation between the local models (31)).

\section{Remark Choice of weighting function dependence}

It is worth emphasising that the weighting functions, $\mu_{i}$, must depend on the same parameter, $\theta$, as the local models in order to ensure consistency across the reconstructed nonlinear system. Inference of the parameter, $\theta$, is of course one outcome of the reconstruction process. This observation is a trivial consequence of the present development, but nevertheless an issue of considerable practical importance (see, for example, the discussion in Johansen \& Murray-Smith (1997)).

\section{CONCLUSIONS}

This paper concerns a fundamental issue in the modelling and realisation of nonlinear systems; namely, whether it is possible to uniquely reconstruct a nonlinear system from a suitable collection of transfer functions and, if so, under what conditions. (Here, 'transfer function' is used as shorthand to denote a linear model based only on measurable input-output data. No restriction to frequency-domain methods is implied or necessary). It is established that

- A family of frozen-parameter linearisations may be associated with a nonlinear LPV/quasi-LPV type of system. While the dynamics of individual members of the family are only weakly related to the dynamics of the nonlinear system, the state-space family of linearisations nevertheless does provide an alternative realisation of the nonlinear system without loss of information. This is, of course, quite different from the situation with classical equilibrium linearisations.

- Knowledge of the input-output dynamics (transfer functions) of the frozen-parameter linearisations of a system is, however, not sufficient to permit reconstruction of the associated nonlinear system. This result is interesting since the state-space frozen- parameter linearisation family does provide a unique representation of a nonlinear system which embodies all of its dynamic characteristics. The difficulty with the transfer function family arises from the degree of freedom available in the choice of state-space realisation of each linearisation.

- Under mild structural conditions, knowledge of a family of augmented transfer functions is sufficient to permit a large class of nonlinear systems to be uniquely reconstructed. That is, the family of augmented transfer functions provides an alternative, and entirely inputoutput based, representation of a nonlinear system. Essentially, the augmented family embodies the information necessary to select state-space realisations for the linearisations which are compatible with one another and with the underlying nonlinear system. The results are constructive, with a state-space realisation of the nonlinear system associated with a transfer function family being obtained as the solution to a number of linear equations.

\section{ACKNOWLEDGEMENTS}

This work was supported through grants from the Royal Society (personal fellowship to DL), Science Foundation Ireland grant 00/PI.1/C067, EU MAC research training network HPRNCT-1999-00107 and EPSRC grant GR/R15863/01. The switching example in section 3 is due to R.Shorten, Hamilton Institute, NUI Maynooth.

\section{REFERENCES}

Johansen, T.A., Murray-Smith,R. (1997) The operating regime approach to nonlinear modelling and Control. Multiple Model Approaches to Modelling \& Control (Ed. Murray-Smith, R., Johansen, T.A.) (Taylor \& Francis, London).

Leith, D.J., Leithead, W.E. (1998a) Appropriate Realisation of MIMO Gain Scheduled Controllers. International Journal of Control, 70, 13-50; (1998b) Gain-Scheduled \& Nonlinear \& Systems: Dynamic Analysis by Velocity-Based Linearisation Families. Int. J. Control, 70, pp289-317;(1998c) GainScheduled Controller Design: An Analytic Framework Directly Incorporating Non-Equilibrium Plant Dynamics. Int. J. Control, 70, pp249-269; (1999) Analytic Framework for Blended Multiple Model Systems Using Linear Local Models. International Journal of Control, 72, 605-619; (2000) Survey of Gain-Scheduling Analysis \& Design. Int. J. Control, 73. pp1001-1025.

Mcloone,S., Irwin,G. (2000) Nonlinear Identification Using Velocity-Based Local Model Networks. Proc. Control 2000 Conf., Cambridge.

Priestley,M.B. (1988) Nonlinear and Nonstationary Time Series. (Academic Press, London).

Reboulet,C., Champetier (1984) A new method for linearising nonlinear systems: the pseudolinearisation. Int. J. Control, 40, 631-638.

Rugh,W.J.(1986) An extended linearisation approach to nonlinear system inversion. IEEE Trans Aut. Control, 31, 725-733.

Shorten,R.N., Narendra,K.S. (1998) Necessary \& Sufficient Conditions for the Existence of a Common Quadratic Lyapunov Function for Two Stable Second Order LTI Systems. Proc. American Control Conf, San Diego. 